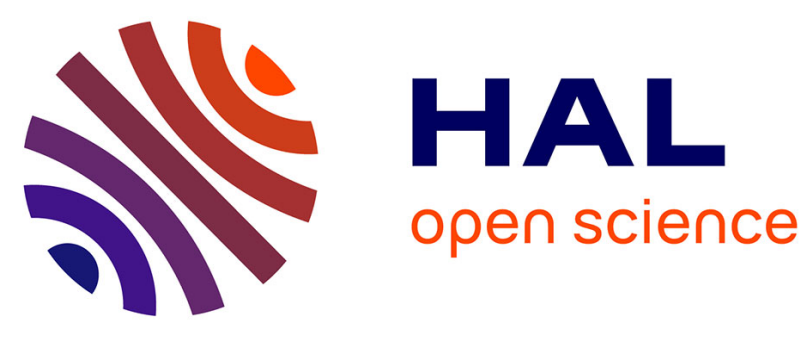

\title{
Un reçu de paiement pour une vente immobilière à terme
}

\author{
Mathieu Tillier, Naïm Vanthieghem
}

\section{To cite this version:}

Mathieu Tillier, Naïm Vanthieghem. Un reçu de paiement pour une vente immobilière à terme. Chronique d'Egypte; bulletin periodique de la Fondation egyptologique reine Elisabeth, 2018, 93 (186), pp.421-431. 10.1484/J.CDE.5.117664 . halshs-02308405

\section{HAL Id: halshs-02308405 https://shs.hal.science/halshs-02308405}

Submitted on 8 Oct 2019

HAL is a multi-disciplinary open access archive for the deposit and dissemination of scientific research documents, whether they are published or not. The documents may come from teaching and research institutions in France or abroad, or from public or private research centers.
L'archive ouverte pluridisciplinaire HAL, est destinée au dépôt et à la diffusion de documents scientifiques de niveau recherche, publiés ou non, émanant des établissements d'enseignement et de recherche français ou étrangers, des laboratoires publics ou privés. 


\title{
Un reçu de paiement pour une vente immobilière à terme
}

\author{
Mathieu Tillier (Sorbonne Université/UMR 8167 Orient et Méditerranée) \\ Naïm Vanthieghem (CNRS/IRHT)
}

Le document que nous éditons ici provient de l'ancienne collection de papiers arabes que le riche amateur d'art G. Michaelidès (1900-1973) $\left({ }^{1}\right)$ acquit dans les années 1920-1930, lorsqu'il vivait en Égypte, et qui entra à sa mort dans les collections de la Bibliothèque universitaire de Cambridge $\left({ }^{2}\right.$ ). Bien que connu leur existence soit connue depuis longtemps, ces papiers et parchemins n'ont que peu retenu l'attention des chercheurs, en comparaison avec des papyrus arabes que le même G. Michaelidès avait patiemment accumulés $\left(^{3}\right)$. Nous proposons ici l'édition de l'un de ces documents, prélude à celle d'autres pièces $\left(^{4}\right)$.

Le document en question est un acte notarié, daté précisément du mois de rağab 447, soit de septembre-octobre 1055. Le texte ne mentionne aucun toponyme ou nisba géographique susceptible de fournir un indice sur sa provenance. La marque d'enregistrement apposée dans le coin supérieur gauche par le cadi - ou le représentant de la justice $\left(^{5}\right)$ - vient heureusement nous éclairer. Son auteur, un certain al-Hasan b. Ibrahīm b. Rizq, est en effet connu pour avoir apposé sa signature dans la marge supérieure d'actes de la famille des Banū Ğirğa $\left(^{6}\right)$, originaire de Țuṭūn dans le sud du Fayoum. Comme les habitants de Ṭuṭun, bourgade à majorité chrétienne, avaient pour habitude de trouver des témoins à Țalìt, qui était majoritairement musulmane et possédait une mosquée et un tribunal $\left({ }^{7}\right)$, il est fort possible

* Les auteurs remercient Lahcen Daaïf pour sa généreuse relecture de cet article et ses suggestions.

(1) Sur ce personnage, cf. W.R. DAWSON, E.C. UPHILL \& M.L. BIERBRIER, Who Was Who in Egyptology (Londres, $1995^{3}$ ), p. 286.

(2) Sur cette collection, S.J. CLACKSON, «The Michaelides Manuscript Collection », ZPE 100 (1994) pp. 223226.

(3) À ce jour, les seules pièces à avoir été publiées sont une pétition fatimide (P. Cambr. UL Inv. Michael. Charta A 169) publiée dans G. KHAN, «A Petition to the Fatimid Caliph al-Āmir », Journal of the Royal Asiatic Society of Great Britain and Ireland (1990), pp. 44-54, un décret mamelouk (P. Cambr. UL Inv. Michael. Charta A 81) paru dans D.S. RICHARDS, «A Mamluk Emir's 'Square' Decree », BSOAS 54 (1991), pp. 63-67 et une série de tarš-s (P. Cambr. UL Inv. Michael. Charta E 28-33) édités par K.R. SCHAEFER, «Eleven Medieval Arabic Block Prints in the Cambridge University Library », Arabica 48 (2001) pp. 210-239.

(4) Nous préparons ainsi l'édition de plusieurs actes juridiques, dont une série de contrats de mariage et divorces qui paraîtront dans un recueil consacré aux mariages et aux séparations dans l'Égypte médiévale.

(5) La qualité de ceux qui ont laissé ces marques n'est jamais mentionnée. À notre connaissance, seul le papier P. Utah Inv. 933 indique que le document a été enregistré par le substitut du juge (al-mustahlaf li-l-hukm) du village de Barbanūda dans le Fayoum; sur ce texte, voir N. VANTHIEGHEM, "L'arabisation des Coptes : un témoin inédit », Egypt and Syria in the Fatimid, Ayyubid and Mamluk Eras VIII. Proceedings of the 19th, 20th, 21st and 22nd International Colloquium Organized at Ghent University in May 2010, 2011, 2012 and 2013 (Louvain, 2016), pp. 121-131.

(6) CPR XXVI 10, 1-2 (Ṭuțūn, 30 rağab 451/11 septembre 1059) ; Chrest. Khoury II 20, 1-2 (Ṭuțūn, 15 ša bān 456/2 août 1064) ainsi que l'inédit P. Lond. Inv. Or. 4684 (11), 2-3 et 22-23 (Ṭuṭun, rağab 455/juin-juillet 1063).

(7) Sur cette question, voir M. TILLIER \& N. VANTHIEGHEM, « La rançon du serment. Un accord à l'amiable au tribunal fatimide de Țalìt », REMMM 140 (2016), pp. 53-72, en part. pp. 59-62. 
qu'al-Hasan b. Ibrahīm b. Rizq ait exercé la fonction de juge au tribunal de Țalìt. C'est sans doute à ce titre qu'il procéda à l'enregistrement d'une transaction dont les protagonistes sont une femme de Țalīt et un homme de Bulğusūq, deux villes voisines de Țuṭūn $\left(^{8}\right)$. Notre document, qui acte une transaction entre deux chrétiens, fut donc vraisemblablement enregistré à Țalīt par des habitants provenant de la ville voisine de Țuṭun.

Un certain Samuel b. Qalamūn reconnaît avoir perçu de Yuhannis b. Damana la somme de deux dinars et demi qu'il lui restait à payer sur le prix de vente de la maison achetée par le second au premier pour une somme totale de six dinars et demi. L'acheteur s'est donc déjà acquitté auparavant de quatre dinars et a rédigé une reconnaissance de dette relative aux deux dinars et demi restant. Maintenant que la somme est payée, Yuhannis b. Damana devient pleinement propriétaire de la maison. L'acte fut dressé devant trois témoins dont les noms et patronymes permettent de penser qu'ils étaient musulmans. Ādam b. Yūsuf b. Wahb, qui apparaît en deuxième position (1.15), était aussi le notaire si l'on en juge par son écriture. Il nota le premier témoignage pour Abū l-Ḥasan 'Īsā, qui devait être analphabète. Le dernier témoin, 'Umar b. 'Alī, manifeste une maîtrise plutôt élémentaire de l'écriture, comme le montre son tracé maladroit, que l'on imagine copié sur les attestations précédentes.

\section{UNE VENTE À TERME}

Contrairement à ce que l'on pourrait croire, le document n'est pas un acte de vente immobilière, qui adopterait un formulaire différent $\left({ }^{9}\right)$, mais un acte établissant que le montant de la transaction a été réglé dans son intégralité. Rédigé sous la forme d'un iqrār («reconnaissance »), il est qualifié de hatt par le rédacteur et premier témoin. Bien que le terme n'apparaisse que dans la seconde moitié (1.9), le document s'apparente à une forme de quittance $\left(\operatorname{bar} \bar{a}^{\prime} a\right)$. Il constitue la dernière pièce d'une transaction dont il rapporte des étapes antérieures. Le notaire mentionne, à la 1. 4, l'établissement (katb) d'une wațīqa, ainsi que la rédaction de ce qu'il appelle un hatt.

Le terme de watīiqa, qui est souvent compris dans le sens générique de «document $»\left({ }^{10}\right)$, prend une acception particulière dans les actes notariés. Dans la Damas des $\mathrm{XI}^{\mathrm{e}}$ et $\mathrm{XII}^{\mathrm{e}}$ siècles,

(8) Chrest. Khoury I 39 (Fayoum, ša bān 456/juillet-août 1064).

(9) Sur le formulaire des actes de ventes immobilières et leurs subdivisions, voir par exemple AL-ȚULAYṬULİ, al-Muqni 'fì 'ilm al-šurūt (Beyrouth : Dār al-kutub al-'ilmiyya, 2000), p. 93 ; AL-SAMARQANDĪ, al-Šurūt wa-l-

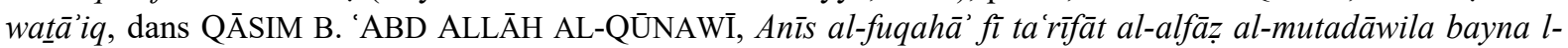
fuqahā', éd. Yaḥyā Murād (Beyrouth : Dār al-kutub al-'ilmiyya, 2004), pp. 20-24 ; IBN ABĪ L-DAM, Kitāb Adab

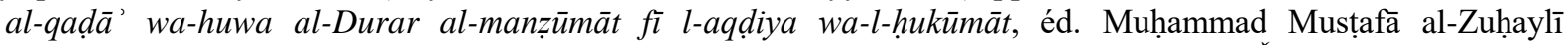
(Beyrouth-Damas : Dār al-fikr al-mu 'āṣir-Dār al-fikr, 1982), pp. 496-517 ; AL-ASYŪṬī, Ğawāhir al- 'uqūd wamu'ìn al-qud̄ăt wa-l-muwaqqi ìn wa-l-šshūd (Le Caire : Maṭba at al-sunna al-muhammadiyya, 1955), I, pp. 7581.

(10) Selon R. Dozy, il peut aussi prendre celui d'« obligation », de «lettre de crédit». R. DOZY, Supplément aux dictionnaires arabes (Leyde, 1881), II, p. 788. 
il apparaît dans des contrats de mariage où il prend le sens d' ' engagement », mais ne désigne pas le document en tant que tel $\left({ }^{11}\right)$. En Égypte, dès le $\mathrm{IV} / \mathrm{X}^{\mathrm{e}}$ siècle, watīiqa s'applique en revanche à un type de document, désigné ainsi dans les marques d'enregistrement ajoutées audessus du texte ou dans les témoignages $\left({ }^{12}\right)$. Une des premières occurrences, remontant à l'an à 304/916-917, s'applique à un acte d'affranchissement d'un esclave; le terme est mis pratiquement sur le même pied que celui de hụğğa $\left({ }^{13}\right)$. La wațīqa semble donc désigner à l'origine une forme d'acte notarié à forte valeur juridique susceptible d'être produit dans un tribunal. Dès le début du $\mathrm{IV}^{\mathrm{e}} / \mathrm{X}^{\mathrm{e}}$ siècle, le mot paraît se spécialiser pour désigner un type particulier, celui d'un acte de vente, ou à tout le moins de transfert de propriété principalement immobilière $\left({ }^{14}\right)$. Il est ainsi utilisé pour désigner un acte de donation charitable, daté de 336/947 et réalisé en faveur des églises de deux monastères $\left({ }^{15}\right)$, et pour l'acte de vente d'une chambre en 339/951 (16).

Tout comme le terme précédent, le mot hațt semble s'appliquer à l'origine, en tant qu'appellation terminologique, à un type documentaire. Il apparait, revêtu d'un sens générique, dans Chrest. Khoury I 41 (Ṭuṭūn, 403/1012-1013), un document où un certain Qāsim b. Ab̄̄ 'Īsā reconnaît que Mīnā b. Ğirğa, le protagoniste des archives des Banū Ğirğa ${ }^{17}$, ne lui doit rien. Dans la clause finale, le texte précise que toute personne qui formulerait une réclamation à l'encontre de Mīnā en la matière verra sa demande déboutée, quelle que soit la forme notariale du document qu'il produirait à l'appui de sa réclamation (1.

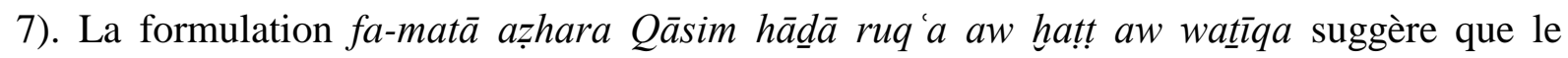
hatt désignait un document dont la forme notariale se situait entre celle d'une ruq 'a, c'est-àdire un simple coupon de papier sans force juridique, et celle d'une wațīqa investie d'une forte valeur légale. C'est ce sens générique que prend notre document dans le témoignage que le notaire Abū l-Ḥasan b. 'İsā a rédigé de sa main. Cependant, à l'époque qui nous occupe, le mot hatt en vient apparemment à désigner aussi, plus spécifiquement, des actes relatifs à des

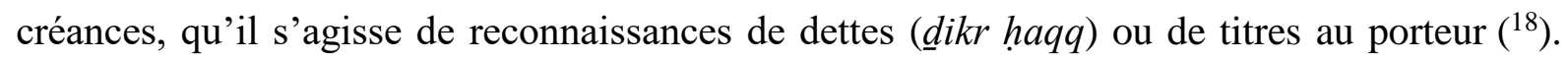
Ce second sens est attesté dans notre document aux 11.5-7, quand le notaire évoque $l^{\prime}$ «obligation » $\left.{ }^{19}\right)$ qui a été établie par le vendeur pour les deux dinars et demi restant à

(11) P. Mariage Séparation 12, $2 ; 24,2$.

(12) Ou encore dans la mention d'un ajout dans le document (lahq). Voir par exemple Chrest. Khoury II 19.

(13) Chrest. Khoury I 22 (304/916).

(14) P. Fahmi Taaqud 8 (an 330/951); P. Fay. Monast. 1 (an 335/946); P. Transmission 2 (an 411/1021); P. Köln Kauf (an 413/1024) ; Chrest. Khoury II 19 (an 417/1026); P. Cair. Arab. I 63 (an 434/1043); Chrest. Khoury II 20 (an 456/1064) ; P. Ardabil 3 (an 567/1172). Le seul acte de vente mobilière que nous ayons trouvé désigné par le terme wațiqa concerne la vente d'une jument. Chrest. Khoury I $54=P$. Vente 25 (an 445/1014).

(15) P. Fay. Monast. 3.

(16) P. Fahmi Taaqud 8.

(17) Y. RAGHEB, «Quelques trésors méconnus de l'Ägyptisches Museum», Forschung in der Papyrussammlung. Eine Festgabe für das Neue Museum (Berlin, 2012), pp. 455-480, en part. pp. 458-459.

(18) Voir par exemple P. Cair. Arab. II $114=$ Chrest. Khoury I 38 (an 241/855-856); CPR XXVI 27 (an 344/956); Chrest. Khoury I 41 (an 403/1012-1013); Chrest. Khoury I 46 (an 454/1062-1063).

(19) Selon le Trésor de la Langue Française (http://atilf.atilf.fr/, consulté le 21/05/2018), une obligation désigne, en droit notarial, un «acte authentique par lequel une personne se reconnaît débitrice envers une autre d'une 
payer sur le prix de la maison vendue.

La watīqa mentionnée dans notre document fait donc vraisemblablement référence à l'acte de vente de la maison en question quand le terme hațt renvoie à une reconnaissance de dette. L'achat de cette maison a ainsi donné lieu, dans un premier temps, à la rédaction d'un acte de vente (watīiqa) mentionnant que le vendeur a reçu de l'acheteur quatre dinars sur un prix de vente s'élevant à six dinars et demi. En complément de cet acte, une obligation (hatț) a été établie, dans laquelle l'acheteur reconnaissait qu'il devait encore deux dinars et demi au vendeur. Quelque temps plus tard, l'acheteur finit par verser ce reliquat au vendeur, qui lui en établit quittance à travers le document que nous éditons ici. Comme ce reçu le mentionne, l'acheteur demande alors au vendeur de lui restituer la reconnaissance de dette afin d'effacer la créance et que le vendeur ne puisse prétendre que le prix de la maison n'a pas été intégralement versé.

Notre document constitue ainsi la dernière pièce notariée d'une vente à terme, un cas qui est prévu par le fiqh. Le juriste hanafite égyptien al-Ṭaḥāwī (m. 321/933) évoque la situation où un vendeur remet (sallama) l'objet de la vente avant d'en avoir reçu le prix total (bi-gayr qaḅ̣ min-hu li-țamani-hā). L'acte de vente, dit-il, doit être rédigé en deux exemplaires comme pour une vente ordinaire; il doit néanmoins mentionner que l'acheteur n'est pas quitte du prix mentionné dans le document (min ġayr an yakūn fulān ya 'ñ̄ al-muštarì bari' min al-taman al-madkūr fì hādā l-kitāb). Par ailleurs, l'acte de vente ne doit pas mentionner la garantie (darak). En effet, explique al-Ṭahāwī, celle-ci oblige le vendeur à restituer le prix de vente à l'acheteur en cas d'impossibilité légale pour ce dernier d'entrer en sa possession. Dans la mesure où le vendeur n'a pas reçu le montant de la vente, il ne peut offrir la garantie qui l'obligerait à le restituer. Plus tard, lorsque l'acheteur acquitte le prix de la vente, il peut demander la rédaction d'une quittance ( $\operatorname{bar}^{\prime}{ }^{\prime} a$ ) établissant qu'il ne doit plus rien au vendeur. Ce document, rédigé sous forme de šahāda (commençant par «Voici ce qu'attestent les témoins mentionnés dans le présent document...»), précise que l'acheteur a réglé le montant total du prix mentionné dans l'acte de vente. C'est, par ailleurs, dans cette quittance que la garantie du vendeur (darak) doit être mentionnée : en échange du prix de vente, le vendeur reconnaît être tenu par l'obligation de garantie $\left({ }^{20}\right)$. Bien qu'il ne soit pas rédigé sous forme de šahāda comme préconisé par al-Ṭaḥāwī, mais sous forme d'iqrār, notre document correspond donc à la quittance qui clôt la vente à terme, établissant que le montant total a été payé et que le vendeur garantit le transfert de propriété.

somme d'argent à titre de prêt ou autre cause, aux conditions arrêtées dans cet acte ».

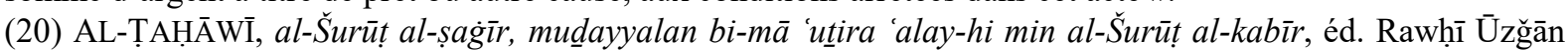
(Bagdad, : Maṭba'at al-'Ānī, 1974), pp. 185-186 ; voir également pp. 188 et 189. 


\section{ÉDITION}

P. Cambr. UL Inv. Michael. Charta B 72

Ṭuṭūn (?) $20,5 \times 17,5 \mathrm{~cm}$ (FIG. 1)

ră̆ab 447/septembre-octobre 1055

Coupon de couleur beige. Le document est complet et toutes ses marges sont conservées, sinon dans les coins supérieurs gauche et droite. Le coupon présente les traces de six plis dans le sens de la hauteur et d'un pli dans le sens de la largeur. Une trace-miroir au niveau de la 1.5 montre que le papier a d'abord été plié en deux dans le sens de la largeur avant d'être plié six fois dans le sens de la hauteur. Le texte est réparti sur onze lignes d'écriture, à quoi s'ajoutent, dans le coin supérieur gauche, la marque d'enregistrement d'un tribunal ainsi que trois témoignages, apposés au bas du coupon et chacun répartis sur deux lignes. L'écriture est cursive et ligaturée, mais néanmoins assez lisible ; quelques lettres sont pourvues de points diacritiques. Au verso, le haut du coupon comporte une série incompréhensible de lettres latines qui ont dû être ajoutées par un marchand d'art peu scrupuleux et désireux de tirer plus d'argent de la pièce qu'il s'apprêtait à vendre.

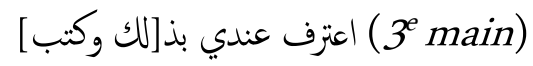

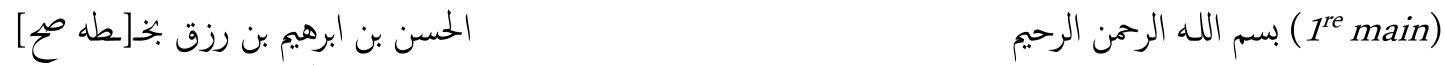

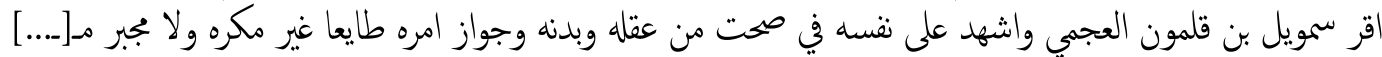

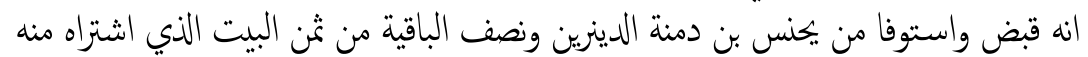

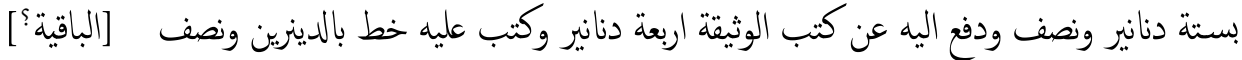

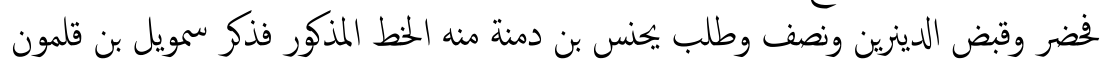

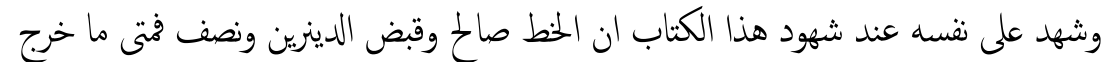

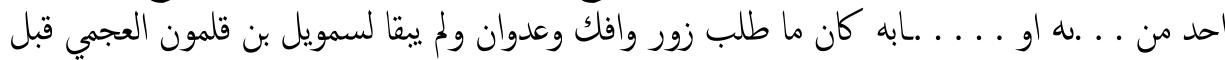

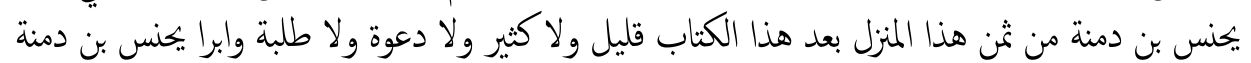

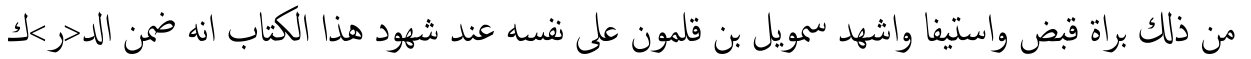

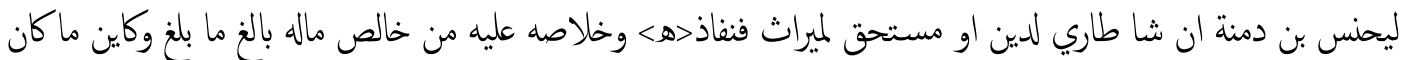

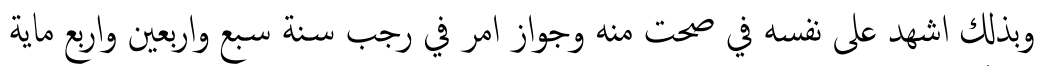

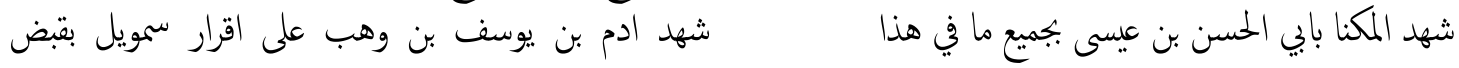

$$
\begin{aligned}
& \text { الباقي } \\
& \text { الذي له من ثن البيت وكتب بخطه في تار كأيخه } \\
& \text { (2 main) } \\
& \text { لكتاب وكتب بخطه }
\end{aligned}
$$

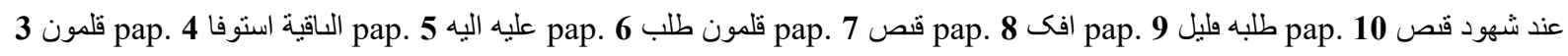
pap. عليه ونعاد 11 pap. اشهد 12 pap. 
«(3e main) Il a reconnu cela en ma présence. Al-Ḥasan b. Ibrāhīm b. Rizq [a écrit de sa main. Valide].

( $1^{\text {re }}$ main) Au nom de Dieu, le Clément, le Miséricordieux. Samuel b. Qalamūn le Copte ( $a l$ - 'ağamī) a avoué et appelé à témoigner contre lui-même, alors qu'il était sain d'esprit et de corps, en pleine capacité, de son plein gré et sans contrainte ni violence [...], qu'il a perçu de Yuhannis b. Damana le paiement intégral des deux dinars et demi qu'il lui restait sur le prix de vente de la maison qu'il lui a achetée, et qui s'élève à six dinars et demi. [Yuhannis b. Damana] lui avait versé à l'établissement de l'acte de vente (katb al-watīiqa), quatre dinars, et avait écrit une obligation (hatte) relative aux deux dinars et demi [restants]. [Samuel b. Qalamūn] s'est présenté, a perçu les deux dinars et demi, et Yuhannis b. Damana lui a demandé [de lui remettre] ladite obligation. Samuel b. Qalamūn a déclaré et témoigné devant les témoins du présent document que l'obligation était valide et a perçu les deux dinars et demi. Si à l'avenir quelqu'un de ... saisit la justice ..., sa réclamation sera considérée comme fausse, mensongère et injuste. Après l'établissement du présent document, Yuhannis b. Damana ne doit plus rien à Samuel b. Qalamūn le Copte au titre de la vente de cette demeure : ce dernier ne pourra lui demander ou lui réclamer ni peu ni prou. Il tient Yuhannis b. Damana quitte de la totalité du montant qui était dû et qu'il a perçu. Samuel b. Qalamūn a appelé à témoigner contre lui-même, devant les témoins du présent document, qu'il offrait à Yuhannis b. Damana garantie de tout vice au cas où surviendrait un débiteur ou l'ayant-droit d'une succession : son exécution et le remboursement lui en incomberaient sur ses propres deniers, quel qu'en soit le montant et la nature. Il a appelé à en témoigner contre lui-même, alors qu'il était sain et en pleine capacité, en rağab de l'an 447.

(1er tém.) Le surnommé Abū l-Ḥasan b. 'Īsā a témoigné de tout ce que contient le présent billet (hat th). Ādam b. Yūsuf a écrit pour lui, à sa demande et en sa présence, à ladite date. $\left(2^{e}\right.$ tém.) Ādam b. Yūsuf b. Wahb a témoigné de l'aveu formulé par Samuel, d'après lequel il a reçu le reliquat qui lui était dû sur le prix de la maison. Il a écrit de sa main à ladite date. ( $2^{e}$ main/3 $3^{e}$ tém.) 'Umar b. 'Alī a témoigné de tout ce que contient le présent document et a écrit de sa main. »

1-2 i tarafa 'indī bi-d̄âllik wa-kataba] | al-Hasan b. Ibrahīm b. Rizq bi-haltți-hi. șaḥha] La restitution est assurée par les parallèles mentionnés dans la n. 6 . On note que le nūn final de l'ism est écrasé au point de ressembler à un $b \bar{a}$ ' ou à l'un de ses homographes; pareille graphie est attestée dans l'inédit P. Lond. Inv. Or. 4684 (11), 22-23. Cette marque signifie que le vendeur, après avoir fait établir la quittance par un notaire, s'est rendu au tribunal pour faire authentifier le document. Sa reconnaissance orale (i tirāf) devant le juge de la justesse des informations portées par le document a été dûment consignée sur l'acte par le juge, ce qui renforce sa valeur légale en cas de contestation ultérieure, en particulier si les témoins viennent à disparaître.

3 Samwīl b. Qalamūn al-ağamī Le terme 'ağamī s'applique en général aux personnes qui ne parlent pas arabe (cf. A. de B. KAZIMIRSKI, Dictionnaire arabe-français [Paris, 1860], I, p. 184); en Égypte, cette nisba est un synonyme du mot qibtī. L'équivalence est notamment assurée par la formule min ašhur al'ağam qui est utilisée à côté de la formule min ašhur al-qibt pour indiquer que les mois de référence mentionnés dans un contrat correspondent au calendrier copte par opposition à celui des musulmans ; sur cette formule, cf. A. GROHMANN, Einführung und Chrestomathie zur arabischen Papyruskunde. I. 
Einführung (Prague, 1954), pp. 230-231.

gayr mukrah wa-lā muğbar $\boldsymbol{m}$. [. . .l On trouve en principe après les participes niés ġayr mukrah wa-lā muğbar les participes positifs tălib ou rāgib, qui sont même parfois associés. On voit cependant clairement après le participe wa-lā muğbar un mìm suivi d'une lettre qui descend sous la ligne - on peut songer par exemple à un $r a \bar{a}$. Il n'est pas impossible que le scribe ait utilisé le participe de la forme II murag்ib au lieu du participe $r a \bar{g} i b$ usuel dans le formulaire. Alternativement, on pourrait proposer de restituer gayr mukrah wa-lā muğbar m [in ahad] (« sans contrainte ni violence de la part de quiconque »).

4 Yuhannis b. Damana Le patronyme est attesté au moins une fois dans P. Prag. Arab. 64, 3. Il s'agit

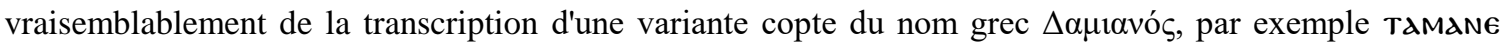
attesté dans P. Ryl. Copt. 165, 2 et P. Lond. Copt. 480.

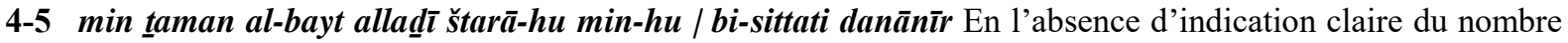
de parts vendues, il faut supposer que c'est l'immeuble en entier qui a changé de propriétaire. Même si le document ne précise pas la taille du bien acheté par Yuhannis b. Damana, le prix de $61 / 2$ dinars paraît cohérent avec ceux pratiqués pour des habitations dans le Fayoum méridional et oriental aux $\mathrm{IV}^{\mathrm{e}}-\mathrm{V}^{\mathrm{e}} / \mathrm{X}^{\mathrm{e}}-\mathrm{XI}^{\mathrm{e}}$ siècles, comme le montre le tableau suivant :

\begin{tabular}{|c|c|c|c|c|c|c|}
\hline Publication & Date & Provenance & Bien vendu & 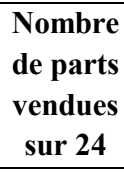 & Prix & $\begin{array}{c}\text { Prix } \\
\text { théorique } \\
\text { pour } 24 \\
\text { parts } \\
\end{array}$ \\
\hline P. Fahmi Taaqud 8 & $339 / 951$ & Bulğusūq & $\dot{g} u r f a$ & 24 parts & 6 dinars & 6 dinars \\
\hline P. Cair. Arab. I 57 & $341 / 952$ & Ṭuṭūn & manzil & 4 parts & 1 dinar & 6 dinars \\
\hline P. Cair. Arab. I 59 & $341 / 952$ & Ṭuṭūn & manzil & 12 parts & 4 dinars & 8 dinars \\
\hline P. Cair. Arab. I 58 & $341 / 952$ & Ṭuṭūn & manzil & 12 parts & $31 / 3$ dinars & $6 \frac{2}{3}$ dinars \\
\hline $\begin{array}{l}\text { P. Frantz-Murphy } \\
\text { Comparison I } 1\end{array}$ & $350 / 961$ & Ṭuṭūn & manzil & 8 parts & 3 dinars & 9 dinars \\
\hline $\begin{array}{l}\text { P. Frantz-Murphy } \\
\text { Comparison I } 2\end{array}$ & $352 / 963$ & Ṭuṭūn & $d \bar{a} r$ & 24 parts & $21 / 6$ dinars & $21 / 6$ dinars \\
\hline P. Fay. Villages 2 & $388 / 998$ & Damuya & bayt & 12 parts & 7 dinars & 14 dinars \\
\hline P. Fay. Villages 3 & $395 / 1004-1005$ & Damuya & $d \bar{a} r$ & 24 parts & 6 dinars & 6 dinars \\
\hline P. Fay. Villages 4 & $399 / 1009$ & Damuya & manzil & 24 parts & 6 dinars & 6 dinars \\
\hline P. Cair. Arab. I 60 & $406 / 1015$ & Bulğusūq & manzil & 24 parts & 5 dinars & 5 dinars \\
\hline P. Fay. Villages 7 & $413 / 1022-1023$ & Damuya & dār qawrā & 24 parts & $21 / 2$ dinars & $2 \frac{1}{2}$ dinars \\
\hline P. Fay. Villages 8 & $413 / 1022-1023$ & Damuya & $\begin{array}{c}\text { manzil }+1 \\
\text { faddān }\end{array}$ & 24 parts & 7 dinars & 7 dinars \\
\hline
\end{tabular}




\begin{tabular}{|c|c|c|c|c|c|c|}
\hline P. Kaufurkunde & $414 / 1024$ & Barbanūda & manzil & $\begin{array}{l}181 / 3 \\
\text { parts }\end{array}$ & $\begin{array}{c}11 / 4^{1 / 8} 1 / 48 \\
\text { dinars }\end{array}$ & $1 \frac{1}{2} 1 / 3$ dinar \\
\hline P. Fay. Villages 18 & $416 / 1025-1026$ & Damuya & dāral-bi'r & ? parts & $23 / 4$ dinars & $?$ \\
\hline P. Utah Inv. 933 & $421 / 1030$ & Barbanūda & manzil & 5 parts & $1 / 21 / 8$ dinar & 3 dinars \\
\hline P. Cair. Arab. I 62 & $429 / 1037$ & Bulğusūq & manzil & 6 parts & $\begin{array}{l}21 / 21 / 4 \\
\text { dinars }\end{array}$ & 11 dinars \\
\hline P. Cair. Arab. I 67 & $450 / 1058$ & Bulğusūq & manzil kabīr & ? parts & 3 dinars & $?$ \\
\hline
\end{tabular}

7 anna l-hatț șālih Le lām de șâliḥ est plus court que ce à quoi on s'attendrait. Une seconde possibilité consisterait à lire șābiḥ (« clair », « manifeste »).

7-8 fa-matā mā harağa |ahad min .... -hi aw . . . a äbi-hi Nous ne sommes pas parvenus à une lecture satisfaisante. On attend ici une clause indiquant qu'au cas où un héritier du vendeur contesterait la vente, sa plainte serait nulle et non avenue. Habituellement, pareille clause commence par les mots matā mā idda'a (cf. par ex. P. Berl. Arab. I 14 et P. Kaufurkunde, 11-12) ou matā mā adraka (cf. par ex. P. Cair. Arab. I, p. 204-206 et P. Cair. Arab. I 72). C'est à notre connaissance la première occurrence, dans une telle formule, du verbe ḩarağa, lequel prend ici le sens de «porter une action en justice ». Après le verbe, on lit clairement le mot ahad suivi de la préposition min, puis sans doute de deux mots coordonnés par $a w$. Du premier, on ne distingue plus qu'un rasm incomplet بوكس, qu'on pourrait être tenté de lire wakilli-hi («son fondé de pouvoir »). Quant au second, son rasm semble se présenter comme suit :

10-11 damina al-da<ra>k| li-Yuhannis b. Damana in šà'a țāri' li-dayn aw mustahiqq li-mīrāt fa-nafādu-

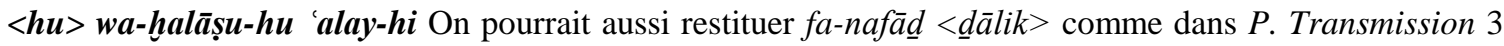
(Uqlūl, an 415/1024) et dans Chrest. Khoury II 20 (Țuṭūn, an 456/1064). Cette expression signifie que le bien immobilier est garanti contre toute hypothèque et toute revendication successorale. Le vendeur s'engage par-là à indemniser l'acquéreur si son titre de propriété était revendiqué par un tiers. Sur cette formule, voir J. A. Wakin, «Darak », EI ${ }^{2}$, XII, p. 198 et W. DIEM, Eine Arabische Kaufurkunde von 1024 n. Chr. aus Ägypten (Wiesbaden, 2004), pp. 43-49.

18-19 'Umar b. 'Alī Un témoin de P. Transmission 3, un document qui provient d'Uqlūl (an 415/1024), porte le nom de 'Umar b. 'Alī b. Hasnūn et est également appelé al-mustahlaf («le substitut »). L'écriture de son nom n'y est pas aussi maladroite. La relative fréquence du nom, l'importante différence chronologique et l'éloignement géographique entre les deux documents laissent par ailleurs penser qu'il ne s'agit pas du même personnage.

\section{CONCLUSION}

Le document que nous éditons témoigne de pratiques transactionnelles qui, jusqu'à présent, n'étaient connues qu'à travers des textes relevant de la théorie juridique. Alors que les actes de vente jusqu'ici édités offrent l'image de transactions assez simples, passant par l'établissement d'un contrat et par la remise du prix total au vendeur, il apparaît que, dans le Fayoum $\mathrm{du} \mathrm{V}^{\mathrm{e}} / \mathrm{XI}^{\mathrm{e}}$ siècle, la vente d'un bien immobilier pouvait suivre un cheminement plus complexe. Un acheteur qui ne disposait pas de la totalité de la somme convenue pouvait contracter une vente à terme et procéder à un versement en plusieurs fois. Chaque étape de la 
transaction donnait lieu à la rédaction de documents qui enregistraient les sommes versées, sous la forme de quittances, et ce qu'il restait à payer. Les obligations consignant les reliquats étaient remises à l'acheteur - peut-être pour être détruites - au fur et à mesure que ce dernier s'en acquittait. L'acheteur pouvait-il, dans un pareil cas, prendre possession de la maison avant d'avoir achevé de la payer, ou devait-il attendre d'avoir transféré la totalité de la somme au vendeur? Le présent document ne répond pas de manière explicite. Aux yeux d'al-Ṭahāwī, cependant, il semble aller de soi que le vendeur remet le bien immobilier à l'acheteur sans attendre d'en avoir reçu le prix total $\left({ }^{21}\right)$. Par ailleurs, le recours à un tel montage juridique suggère qu'il profitait aux deux parties contractantes. L'opération s'apparentait peut-être à une forme de crédit hypothécaire, l'acheteur pouvant, en contrepartie d'un prix plus élevé que celui du marché, jouir immédiatement du bien qu'il était en train d'acquérir.

Cette quittance de vente à terme permet en outre de mieux comprendre la terminologie employée par les documents juridiques pour parler d'eux-mêmes. Les termes wațīqa et hațt, tous deux employés dans le texte, apparaissent comme des catégories distinctes. Le vocable watīiqa semble avoir à l'origine désigné un type assez général d'acte juridique susceptible d'être produit devant un tribunal, avant de se restreindre, à partir $\mathrm{du} \mathrm{IV}^{\mathrm{e}} / \mathrm{X}^{\mathrm{e}}$ siècle, à un acte relatif à un transfert de propriété. Le mot hatțt, terme générique à l'origine, en vint souvent à désigner plus spécifiquement une obligation dans le cadre d'une créance.

Notre document vient enfin éclairer l'articulation entre transactions privées et institutions judiciaires dans le Fayoum du $\mathrm{V}^{\mathrm{e}} / \mathrm{XI}^{\mathrm{e}}$ siècle. Les deux parties contractantes, apparemment des chrétiens, firent établir la quittance par un notaire et des témoins musulmans, puis l'enregistrèrent auprès d'une institution judiciaire musulmane, peut-être le tribunal de Ṭalīt connu par d'autres sources. Le document offre ainsi une nouvelle confirmation de l'importance prise par ce tribunal musulman aux yeux de populations chrétiennes qui avaient tout intérêt à y faire valider leurs actes afin de se prémunir contre des contestations ultérieures devant la même institution.

Sorbonne Université

CNRS-IRHT (Paris)
Mathieu TILLIER

Naïm VANTHIEGHEM

(21) Voir supra. 


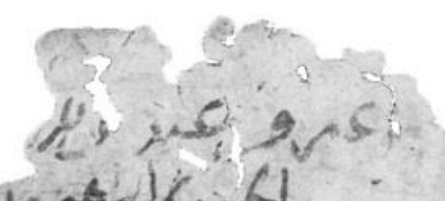

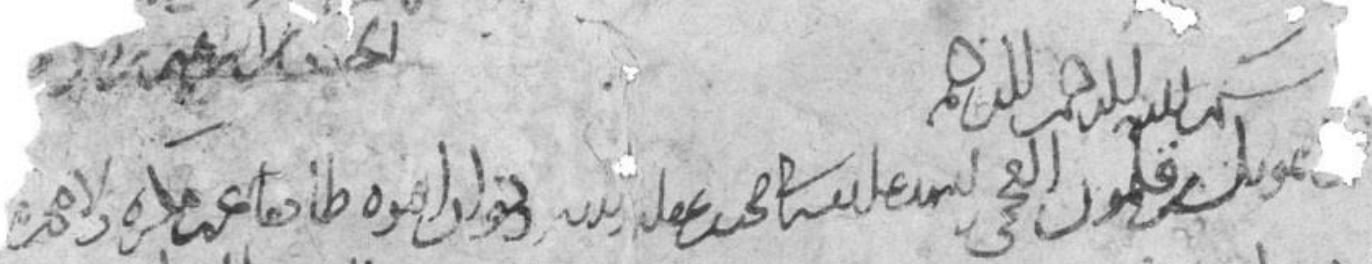

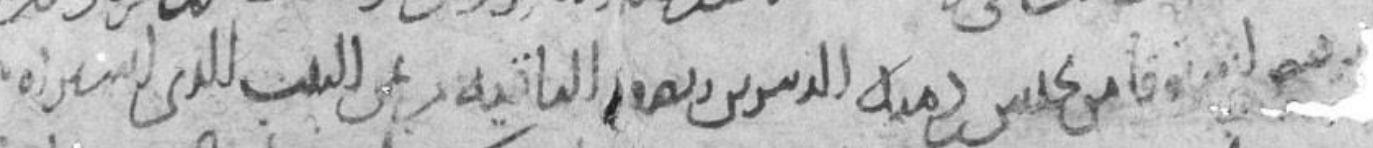

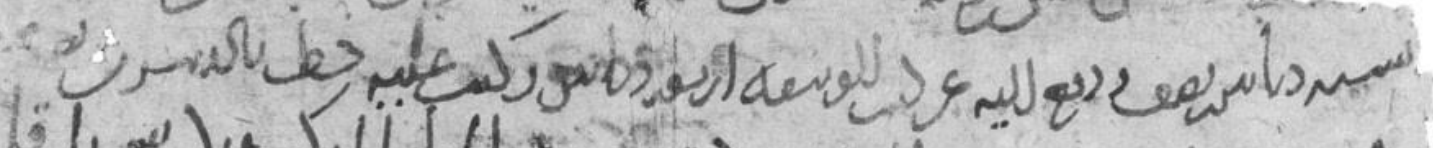
U.

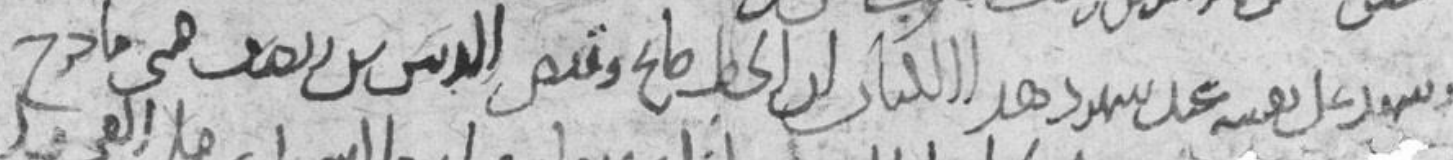

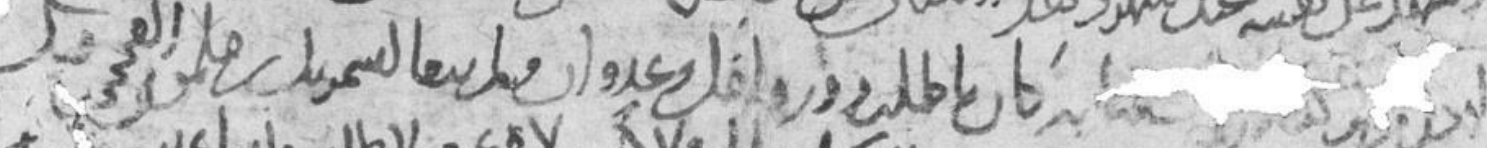

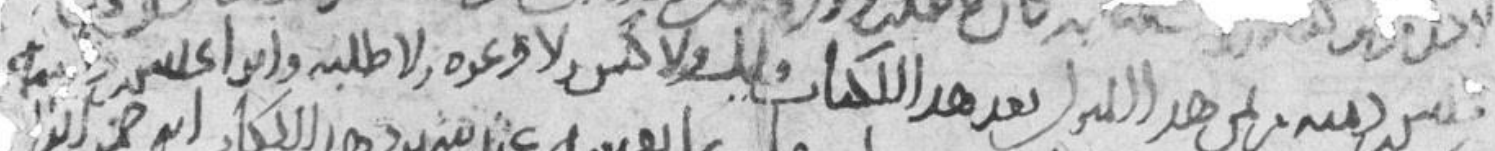

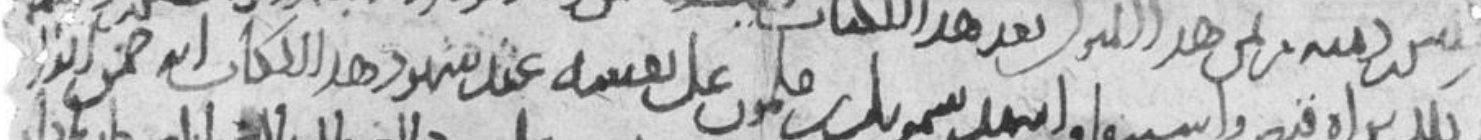

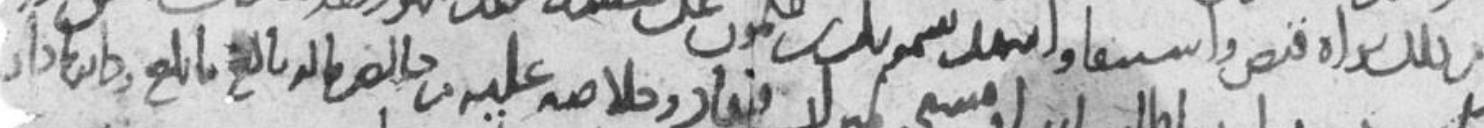

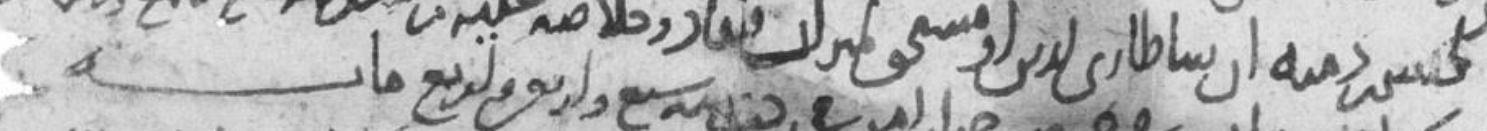

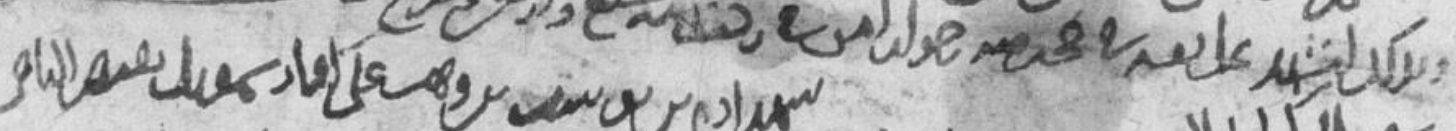

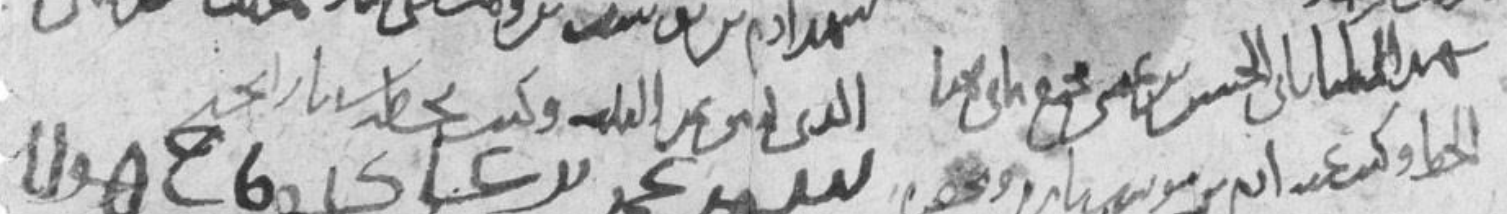
ais

Fig. 1 : P. Cambr. UL Inv. Michael. Charta B 72 recto (C) Cambridge University Library) 